\title{
Analysis on Clinical Characteristics and Influencing Factors of Patients with Locoregionally Advanced Nasopharyngeal Carcinoma
}

\author{
Wei Zheng ${ }^{1 \&}$, Yuan-Ji Xu ${ }^{2 \&}$, Su-Fang Qiu${ }^{1}$, Jing-Feng Zong ${ }^{1}$, Ling-Ling Huang ${ }^{2}$, \\ Chao-Bin Huang ${ }^{1}$, Shao-Jun Lin ${ }^{1}$, Jian-Ji Pan ${ }^{1,2 *}$
}

\begin{abstract}
Background: To explore the independent prognostic factors for the recurrence/metastasis of patients with locoregionally advanced nasopharyngeal carcinoma (LANPC). Materials and Methods: A total of 604 patients initially diagnosed as LANPC by pathohistology in Fujian Provincial Cancer Hospital were selected to analyze the relationship between the clinical pathological patterns, therapeutic protocols and clinical stages with the recurrence/metastasis of LANPC. Results: The 1-,3- and 5-year locoregionally recurrent rates of LANPC patients were $2.0 \%, 9.5 \%$ and $12.9 \%$ respectively, with average recurrent period being 78 months. Univariate analysis results indicated that clinical stages had certain influence on the recurrent period of LANPC patients. However, COX regression models showed that ages, genders and clinical stages were not the independent prognostic factors influencing the recurrence. The 1-, 3- and 5-year metastatic rates of LANPC patients were $6.6 \%, 17.5 \%$ and $18.8 \%$ respectively, with average metastatic period of 73 months. Univariate analysis results demonstrated that ages, $\mathrm{N}$ stages, clinical stages, locations of lymph node, retropharyngeal lymph node and extracapsular invasion of lymph node had certain influence on the metastatic period of LANPC patients. Additionally, further COX regression analysis results suggested that $T$ stages, reduction protocols and extracapsular invasion of lymph node were the independent prognostic factors influencing the metastasis of patients with LANPC, in which T stages and extracapsular invasion of lymph node were the pestilent factors while reduction protocols the protective factor. Conclusions: Induction chemotherapy is beneficial to LANPC patients with initial treatment, and the metastatic rate decreases greatly after the application of reduction chemotherapy.
\end{abstract}

Keywords: Locoregionally advanced asopharyngeal carcinoma - induction chemotherapy - radioptherapy - recurrence

Asian Pac J Cancer Prev, 16 (10), 4393-4399

\section{Introduction}

Nasopharyngeal carcinoma (NPC) is one of the most common head-neck malignant tumors in China, with especially high morbidity in the south region. The morbidity of NPC in Guangdong Province ranks the third in all malignant tumors and the first in head-neck tumors (Hu et al., 2014; Li et al., 2014), and with significant racial, regional and family aggregation phenomenon $(\mathrm{Qu}$ et al., 2015). Radiotherapy is the primary therapeutic method for locoregional advanced NPC (LANPC) because LANPC is sensitive to radiotherapy. In recent years, with the wide application of intensity modified radiation therapy (IMRT) and comprehensive therapies, the therapeutic efficacy for NPC patients has been further improved and the 5-year local recurrence free survival rate is up to $78.4 \% \sim 80 \%$ (Lin et al., 2014; Lee et al., 2014; Wu et al., 2014). However, the therapeutic efficacy for LANPC patients is still unsatisfactory and the 5-year survival rate is only $70 \%$ (Wu et al., 2014). Recurrence and metastasis are commonly seen after the initial treatment of LANPC, which can lead to the failure rate of first-cycle radiotherapy up to $25 \%$. Moreover, distant metastasis is the primary failure model, accounting for $53.6 \%$ 63.1\% of the whole recurrence, followed by the locoregional recurrence and regional lymph node metastasis (Chen et al., 2013; Zeng et al., 2014). At present, radiochemotherapy is still the major protocol for the treatment of LANPC patients, and in order to further improve its therapeutic efficacy, the independent prognostic factors influencing the therapeutic failure models should be firstly explored. Therefore, this study analyzed the clinical data of 604 LANPC patients and explored the relationship between clinical pathological characteristics and therapeutic protocols with the LANPC recurrence/metastasis, aiming to search the independent risk factors for LANPC patients with recurrence/metastasis and predicate the clinical efficacy of secondary-cycle treatment after recurrence, so as to 
provide theoretical basis for the establishment of standard therapeutic protocols for patients with LANPC.

\section{Materials and Methods}

\section{General data}

This study was approved by the Ethics Committee of Fujian Provincial Cancer Hospital and all informed consent forms were signed by the patients and (or) their families. Totally 604 patients initially diagnosed as LANPC without metastasis who were admitted in Fujian Provincial Cancer Hospital from June, 2005 to October, 2007 were selected. All patients were confirmed by pathology and classified into stage III and IV according to the Union for International Cancer Control (UICC)/ American Joint Committee on Cancer (AJCC) criteria for NPC (the 7th version). There were 466 males and 138 females, in which 395 were $\leq 50$ years and $209>50$ years; 575 were with squamous carcinoma and 29 with nonsquamous carcinoma; $\mathrm{T}$ stages: 48 in stage T1, 61 in T2, 342 in T3 and 153 in T4; N stages: 49 in stage N0, 248 in $\mathrm{N} 1,247$ in N2 and 60 in N3; clinical stages: 401 in stage III, and 203 in stage IV; locations of lymphadenovarix: 165 patients without lymphadenovarix, 347 on upper neck, 67 on lower neck and 25 on clavicle; 476 were with retropharyngeal lymph node; and 370 were with extracapsular invasion of lymph node.

\section{Methods}

Evaluation before treatment: Evaluation before treatment included record analysis and physical examination, the latter of which was consisted of blood routine, nasopharyngoscope, magnetic resonance image (MRI) on nasopharynx and neck, chest $\mathrm{X}$ ray or CR scan, abdominal color ultrasound or MRI, electrocardiogram and emission CT (ECT) bone image. Partial patients also needed examination with positron emission computed tomography (ECT), which was decided by the doctorin-charge according to the patients' practical conditions.

Initial therapeutic outcome: Radical radiotherapy was given to all patients, in which 399 with routine radiotherapy and 205 with IMRT; the total radiotherapeutic dosage on nasopharynx was 66.00 85.60 Gy with average one of (70.87 \pm 3.10$) \mathrm{Gy}$, and ratiotherapeutic times were 30 40 times with average ones being $(34.01 \pm 2.38)$ times; the maximum radiotherapeutic dosage for lymph node was $50.00 \sim 79.00$ Gy with average one of $(65.44 \pm 5.19) \mathrm{Gy}$, and the radiotherapeutic times for neck were 25 39 times with average ones being $(32.00 \pm 2.46)$ times. The detailed techniques referred to the previously reported research by Cancer Hospital of Fujian Medical University (Lin et al., 2009; Xu et al., 2010). Additionally, emergency treatments, including surgery, dimensional conformal radiation therapy (3D-CRT), chemotherapy, intracavitary brachytherapy and intensity modulated radiation therapy (IMRT) were given to patients who had persistent or relapse diseases.

Of all patients, 549 received chemotherapy, in which 153 were treated with recurrent chemotherapy, 104 with 1 -cycle chemotherapy and 49 with $>2$-cycle chemotherapy. The administrative protocols included: i) cisplatin, $80 \sim 100 \mathrm{mg} / \mathrm{m}^{2}, \mathrm{~d} 1 \sim \mathrm{d} 3,21 \mathrm{~d}$ as a cycle; ii) paclitaxel, $135 \mathrm{mg} / \mathrm{m}^{2}, \mathrm{~d} 1$ and cisplatin, $80 \sim 100 \mathrm{mg} / \mathrm{m}^{2}$, $\mathrm{d} 1 \sim \mathrm{d} 3,21 \mathrm{~d}$ as a cycle; iii) gemcitabine, $1000 \mathrm{mg} / \mathrm{m}^{2}, \mathrm{~d} 1$ and $\mathrm{d} 8$ and cisplatin, $80 \sim 100 \mathrm{mg} / \mathrm{m}^{2}, \mathrm{~d} 1 \sim \mathrm{d} 3,21 \mathrm{~d}$ as a cycle; $i v$ ) fluorouracil, $800 \mathrm{mg} / \mathrm{m}^{2}, \mathrm{~d} 1 \sim \mathrm{d} 5$ and cisplatin, $80 \sim 100 \mathrm{mg} / \mathrm{m}^{2}, \mathrm{~d} 1 \sim \mathrm{d} 3,21 \mathrm{~d}$ as a cycle. The detailed chemotherapeutic cycles and administrative protocols were determined by the doctor-in-charge according to the practical conditions of patients. A total of 520 patients received induction chemotherapy, in which 36 received 1-cycle and $484>2$-cycle chemotherapy. The intervals of induction chemotherapy were 2 weeks, and radiotherapy was conducted within 1 week after the second-cycle induction chemotherapy. Induction protocols: 444 patients were treated with paclitaxel plus platinum, 13 with gemcitabine plus platinum and 63 with fluorouracil plus platinum, whose administrative dosage and times were the same with those of concurrent chemotherapy. Moreover, 98 patients were treated with adjuvant cisplatin-based chemotherapy accepting the advices of the attending radiation oncologists.

Follow up: The median follow-up period was 65 months (3 86 months) in this study. The primary endpoint of the follow up was overall survival (OS) and second endpoints were progression-free survival (PFS), locoregional free survival (LRFS) and distant metastasisfree survival (DMFS). During the treatment, all patients were evaluated 1 time/week. After treatment, they were followed up 1 time/3 months within the first 2 years, and 1 time/ 6 months in the next 3 years and then 1 time/year every year.

\section{Observational indexes}

The recurrence and metastasis were observed to analyze the relevance between recurrence/metastasis with clinical pathological characteristics and therapeutic protocols. The periods of recurrence and metastasis were recorded to analyze the influence of each factor on recurrence/metastasis of LANPC patients.

\section{Statistical data analysis}

Double input of data obtained after follow up was performed with EpiData 3.0. SPSS 17.0 and SAS 9.3 software were applied for all data analysis. As to categorical variables, the frequency and percentage of each variable were calculated and detected by $\chi^{2}$ test or continuous correction $\chi^{2}$ test whereas rank categorical variables were detected with $\chi^{2}$ trend test. Life table was applied to calculate the 1-, 3- and 5-year DMFS and LRFS and Log-Rank test was adopted to compare the differences among groups. COX stepwise regression analysis was used to determine the relevant factors influencing the recurrence and metastasis of LANPC. $P<0.05$ was considered to be statistically significant.

\section{Results}

Analysis on relevance between recurrence and each factor

The recurrent rate was evidently lower in patients in clinical stage III than those in IV $(P<0.05)$ and was apparently lower in survival patients than those died 
$(P<0.01)$; the different $\mathrm{T}$ stages were in close association with recurrence $(P<0.05)$; but there was no significant difference between the rest factors and recurrence $(P>0.05)$ (Table 1).

\section{Influence of each factor on recurrent time}

The life table method was applied to analyze the follow-up data of all patients, and the results indicated that the 1-, 3- and 5-year recurrent rates of LANPC patients were $2.0 \%, 9.5 \%$ and $12.9 \%$ respectively, with average recurrent period being 78 months $(95 \%$ confidence intervals: 76.43 79.86 months).

In order to observe the influence of each factor on recurrent period, log-rank test was adopted, in which ages, pathological patterns, $\mathrm{T}$ stages, $\mathrm{N}$ stages, concurrent chemotherapy, locations of lymphadenovarix, retropharyngeal lymph node, chemotherapeutic cycles, induction chemotherapy and the induction protocols were excluded from the test due to their values, while the rest factor results were shown in Table 2. It was suggested in the table that clinical patterns and death had certain influence on the recurrent period of LANPC patients whereas the rest factors had no impact.

On this basis, genders, ages, pathological patterns, $\mathrm{T}$ stags, $\mathrm{N}$ stags, radiotherapeutic techniques, concurrent chemotherapy, locations of lymphadenovarix, induction chemotherapeutic protocols, retropharyngeal lymph node, chemotherapeutic cycles, extracapsular invasion of lymph node and metastasis were set as the variates to conduct the multivariate analysis with COX risk ratio regression models (clinical stages were excluded from the analysis due to the high association with TNM stages). It was believed that the above factors were not the independent prognostic factors influencing the recurrence of LANPC because there was no significant difference according to the terminal COX regression equation $\left(\chi^{2}=23.758\right.$, $P=0.126)$.

Table 1. Analysis on Relevance between Recurrence and Each Factor

\begin{tabular}{|c|c|c|c|c|c|c|}
\hline Influencing factors & Witho & $\begin{array}{l}\text { out recurrence } \\
\text { (n) }\end{array}$ & $\begin{array}{l}\text { With recurrence } \\
\text { (n) }\end{array}$ & $\mathrm{N}$ & $\chi^{2}$ value & $P$ value \\
\hline \multirow[t]{2}{*}{ Gender } & Male & 408 & 58 & 466 & 0.249 & 0.618 \\
\hline & Female & 123 & 15 & 138 & & \\
\hline \multirow[t]{2}{*}{ Ages (years) } & $\leq 50$ & 348 & 47 & 395 & 0.038 & 0.846 \\
\hline & $>50$ & 183 & 26 & 209 & & \\
\hline \multirow[t]{2}{*}{ Pathological patterns } & Squamous carcinoma & 507 & 68 & 575 & 0.338 & 0.561 \\
\hline & Non-squamous carcinoma & a 24 & 5 & 29 & & \\
\hline \multirow[t]{4}{*}{$\mathrm{T}$ stages } & 1 & 43 & 5 & 48 & 9.035 & 0.029 \\
\hline & 2 & 52 & 9 & 61 & & \\
\hline & 3 & 311 & 31 & 342 & & \\
\hline & 4 & 125 & 28 & 153 & & \\
\hline \multirow[t]{4}{*}{$\mathrm{N}$ stages } & 0 & 47 & 2 & 49 & 3.591 & 0.309 \\
\hline & 1 & 216 & 32 & 248 & & \\
\hline & 2 & 217 & 30 & 247 & & \\
\hline & 3 & 51 & 9 & 60 & & \\
\hline \multirow{2}{*}{ Clinical stages } & III & 362 & 39 & 401 & 6.256 & 0.012 \\
\hline & IV & 169 & 34 & 203 & & \\
\hline \multirow[t]{2}{*}{ Radiotherapeutic techniques } & Routine radiotherapy & 346 & 53 & 399 & 1.586 & 0.208 \\
\hline & IMRT & 185 & 20 & 205 & & \\
\hline \multirow{2}{*}{ Concurrent chemotherapy } & No & 399 & 52 & 451 & 0.518 & 0.472 \\
\hline & Yes & 132 & 21 & 153 & & \\
\hline \multirow[t]{4}{*}{ Locations of lymphadenovarix } & No & 147 & 18 & 165 & 2.422 & 0.490 \\
\hline & Upper neck & 303 & 44 & 347 & & \\
\hline & Lower neck & 61 & 6 & 67 & & \\
\hline & Superclavical & 20 & 5 & 25 & & \\
\hline \multirow[t]{4}{*}{ Induction chemotherapeutic protocols } & No & 76 & 8 & 84 & 6.791 & 0.079 \\
\hline & Paclitaxel+platinum & 395 & 49 & 444 & & \\
\hline & Gemcitabine+platinum & 10 & 3 & 13 & & \\
\hline & Fluorouracil+platinum & 50 & 13 & 63 & & \\
\hline \multirow[t]{2}{*}{ Retropharyngeal lymph node } & No & 114 & 14 & 128 & 0.202 & 0.653 \\
\hline & Yes & 417 & 59 & 476 & & \\
\hline \multirow[t]{2}{*}{ Chemotherapeutic cycle } & $\leq 2$ cycles & 372 & 46 & 418 & 1.494 & 0.222 \\
\hline & $\geq 3$ cycles & 159 & 27 & 186 & & \\
\hline \multirow[t]{2}{*}{ Extracapsular invasion of lymph node } & No & 208 & 26 & 234 & 0.342 & 0.559 \\
\hline & Yes & 323 & 47 & 370 & & \\
\hline \multirow[t]{5}{*}{ Lost to follow-up } & No & 494 & 69 & 563 & 0.031 & 0.861 \\
\hline & Yes & 37 & 4 & 41 & & \\
\hline & Death & & & & & \\
\hline & Survival & 401 & 24 & 425 & 55.959 & $<0.0001$ \\
\hline & Died & 130 & 49 & 179 & & \\
\hline \multirow[t]{2}{*}{ Metastasis } & No & 425 & 64 & 489 & 2.426 & 0.119 \\
\hline & Yes & 106 & 9 & 115 & & \\
\hline
\end{tabular}


Wei Zheng et al

Table 2. Univariate Analysis on Influence of Each Factor on Recurrent Time

\begin{tabular}{|c|c|c|c|c|c|}
\hline Influencing factors & $\begin{array}{c}\text { 1-year recurrent rate } \\
(\%)\end{array}$ & $\begin{array}{c}3 \text {-year recurrent rate } \\
(\%)\end{array}$ & $\begin{array}{c}5 \text {-year recurrent rate } \\
(\%)\end{array}$ & $\chi^{2}$ value & $P$ value \\
\hline General & 2.0 & 9.5 & 12.9 & - & - \\
\hline \multicolumn{6}{|l|}{ Gender } \\
\hline Male & 1.9 & 10.5 & 13.2 & \multirow[t]{2}{*}{0.501} & \multirow[t]{2}{*}{0.479} \\
\hline Female & 2.2 & 6.1 & 12.0 & & \\
\hline \multicolumn{6}{|l|}{ Clinical stages } \\
\hline III & 1.3 & 7.7 & 10.4 & \multirow[t]{2}{*}{8.411} & \multirow[t]{2}{*}{0.004} \\
\hline IV & 3.5 & 13.1 & 18.3 & & \\
\hline \multicolumn{6}{|l|}{ Radiotherapeutic techniques } \\
\hline Routine radiotherapy & 2.3 & 10.9 & 14.4 & \multirow[t]{2}{*}{2.100} & \multirow[t]{2}{*}{0.147} \\
\hline IMRT & 1.5 & 6.6 & 10.2 & & \\
\hline \multicolumn{6}{|c|}{ Extracapsular invasion of lymph node } \\
\hline Yes & 2.5 & 11.0 & 13.4 & \multirow[t]{2}{*}{1.010} & \multirow[t]{2}{*}{0.315} \\
\hline No & 1.3 & 7.1 & 12.0 & & \\
\hline \multicolumn{6}{|l|}{ Death } \\
\hline Survival & 0.0 & 2.1 & 5.1 & \multirow[t]{2}{*}{129.815} & \multirow[t]{2}{*}{$<0.001$} \\
\hline Died & 7.0 & 33.0 & 40.4 & & \\
\hline
\end{tabular}

Table 3. Relevance between Metastasis and Each Factor

\begin{tabular}{|c|c|c|c|c|c|c|}
\hline Influencing factors & With & $\begin{array}{l}\text { out metastasis } \\
\qquad(\%)\end{array}$ & $\begin{array}{c}\text { With metastasis } \\
(\%)\end{array}$ & $\mathrm{N}$ & $\chi^{2}$ value & $P$ value \\
\hline \multirow[t]{2}{*}{ Gender } & Males & 369 & 97 & 466 & 4.172 & 0.041 \\
\hline & Females & 120 & 18 & 138 & & \\
\hline \multirow[t]{2}{*}{ Ages } & $\leq 50$ & 326 & 69 & 395 & 1.829 & 0.176 \\
\hline & $>50$ & 163 & 46 & 209 & & \\
\hline \multirow[t]{2}{*}{ Clinical patterns } & Squamous carcinoma & 468 & 107 & 575 & 1.444 & 0.230 \\
\hline & Non-squamous carcinoma & 21 & 8 & 29 & & \\
\hline \multirow[t]{4}{*}{ T stages } & 1 & 38 & 10 & 48 & 7.7481 & 0.052 \\
\hline & 2 & 53 & 8 & 61 & & \\
\hline & 3 & 285 & 57 & 342 & & \\
\hline & 4 & 113 & 40 & 153 & & \\
\hline \multirow[t]{4}{*}{$\mathrm{N}$ stages } & 0 & 47 & 2 & 49 & 11.758 & 0.008 \\
\hline & 1 & 206 & 42 & 248 & & \\
\hline & 2 & 192 & 55 & 247 & & \\
\hline & 3 & 44 & 16 & 60 & & \\
\hline \multirow[t]{2}{*}{ Clinical stages } & III & 339 & 62 & 401 & 9.911 & 0.002 \\
\hline & IV & 150 & 53 & 203 & & \\
\hline \multirow[t]{2}{*}{ Raditherapeutic techniques } & Routine radiotherapy & 324 & 75 & 399 & 0.045 & 0.832 \\
\hline & IMRT & 165 & 40 & 205 & & \\
\hline \multirow[t]{2}{*}{ Concurrent chemotherapy } & No & 368 & 83 & 451 & 0.468 & 0.494 \\
\hline & Yes & 121 & 32 & 153 & & \\
\hline \multirow[t]{4}{*}{ Locations of lymphadenovarix } & No & 148 & 17 & 165 & 12.392 & 0.006 \\
\hline & Upper neck & 273 & 74 & 347 & & \\
\hline & Lower neck & 50 & 17 & 67 & & \\
\hline & Superclavical & 18 & 7 & 25 & & \\
\hline \multirow[t]{4}{*}{ Induction chemotherapeutic protocols } & No & 64 & 20 & 84 & 3.703 & 0.295 \\
\hline & Paclitaxel+platinum & 365 & 79 & 444 & & \\
\hline & Gemcitabine+platinum & 12 & 1 & 13 & & \\
\hline & Fluorouracil+platinum & 48 & 15 & 63 & & \\
\hline \multirow[t]{2}{*}{ Retropharyngeal lymph node } & No & 115 & 13 & 128 & 8.315 & 0.004 \\
\hline & Yes & 374 & 102 & 476 & & \\
\hline \multirow[t]{2}{*}{ Chemotherapeutic cycle } & $\leq 2$ cycles & 343 & 75 & 418 & 1.060 & 0.303 \\
\hline & $\geq 3$ cycles & 146 & 40 & 186 & & \\
\hline \multirow[t]{2}{*}{ Extracapsular invasion of lymph node } & No & 208 & 26 & 234 & 15.578 & $<0.001$ \\
\hline & Yes & 281 & 89 & 370 & & \\
\hline \multirow[t]{2}{*}{ Lost to follow-up } & No & 451 & 112 & 563 & 3.921 & 0.048 \\
\hline & Yes & 38 & 3 & 41 & & \\
\hline \multirow[t]{2}{*}{ Death } & Survival & 407 & 18 & 425 & 203.903 & $<0.001$ \\
\hline & Died & 82 & 97 & 179 & & \\
\hline \multirow[t]{2}{*}{ Metastasis } & No & 425 & 106 & 531 & 2.426 & 0.119 \\
\hline & Yes & 64 & 9 & 73 & & \\
\hline
\end{tabular}


DOI:http://dx.doi.org/10.7314/APJCP.2015.16.10.4393 Analysis on Clinical Characteristics and Influencing Factors of Patients with Locoregionally Advanced Nasopharyngeal Carcinoma

Table 4. Univariate Analysis of Each Factor on Metastatic Period

\begin{tabular}{|c|c|c|c|c|c|}
\hline Influencing factors & $\begin{array}{c}\text { 1-year metastatic rate } \\
(\%)\end{array}$ & $\begin{array}{c}\text { 3-year metastatic rate } \\
(\%)\end{array}$ & $\begin{array}{c}\text { 5-year metastatic rate } \\
(\%)\end{array}$ & $\chi^{2}$ value & $P$ value \\
\hline General & 6.6 & 17.5 & 18.8 & - & - \\
\hline \multicolumn{6}{|l|}{ Gender } \\
\hline Males & 7.5 & 19.6 & 20.6 & \multirow[t]{2}{*}{4.578} & \multirow{2}{*}{0.032} \\
\hline Females & 3.6 & 10.4 & 12.8 & & \\
\hline \multicolumn{6}{|l|}{ Ages } \\
\hline$\leq 50$ years & 5.3 & 15.9 & 16.7 & \multirow[t]{2}{*}{2.878} & \multirow[t]{2}{*}{0.090} \\
\hline$>50$ years & 9.2 & 20.5 & 22.9 & & \\
\hline \multicolumn{6}{|l|}{ Pathological patterns } \\
\hline Squamous carcinoma & 6.5 & 17.1 & 18.3 & \multirow[t]{2}{*}{1.659} & \multirow[t]{2}{*}{0.198} \\
\hline Non-squamous carcinoma & 10.3 & 24.3 & 28.3 & & \\
\hline \multicolumn{6}{|l|}{$\mathrm{N}$ stages } \\
\hline 0 & 0.0 & 2.2 & 4.6 & \multirow[t]{4}{*}{12.182} & \multirow[t]{4}{*}{0.007} \\
\hline 1 & 4.8 & 14.7 & 16.5 & & \\
\hline 2 & 6.5 & 20.5 & 21.8 & & \\
\hline 3 & 20.0 & 26.8 & 26.8 & & \\
\hline \multicolumn{6}{|l|}{ Clinical stages } \\
\hline III & 4.5 & 14.2 & 15.6 & \multirow[t]{2}{*}{11.972} & \multirow[t]{2}{*}{$<0.001$} \\
\hline IV & 10.9 & 24.0 & 25.1 & & \\
\hline \multicolumn{6}{|l|}{ Locations of lymphadenovarix } \\
\hline No & 1.8 & 9.5 & 10.9 & \multirow[t]{4}{*}{12.874} & \multirow[t]{4}{*}{0.005} \\
\hline Upper neck & 6.7 & 18.8 & 20.5 & & \\
\hline Lower neck & 12.0 & 26.0 & 26.0 & & \\
\hline Superclavical & 24.0 & 28.2 & 28.2 & & \\
\hline \multicolumn{6}{|l|}{ Retropharyngeal lymph node } \\
\hline Yes & 8.2 & 19.7 & 21.1 & \multirow[t]{2}{*}{7.680} & \multirow[t]{2}{*}{0.006} \\
\hline No & 0.8 & 9.1 & 10.1 & & \\
\hline \multicolumn{6}{|c|}{ Extracapsular invasion of lymph node } \\
\hline Yes & 9.5 & 22.1 & 23.7 & \multirow[t]{2}{*}{16.255} & \multirow[t]{2}{*}{$<0.001$} \\
\hline No & 2.1 & 10.1 & 11.1 & & \\
\hline \multicolumn{6}{|l|}{ Death } \\
\hline Survival & 0.9 & 2.6 & 3.3 & \multirow[t]{2}{*}{301.451} & $<0.001$ \\
\hline Died & 20.4 & 57.5 & 62.3 & & \\
\hline Induction chemotherapy & & & & & \\
\hline Yes & 6.0 & 16.6 & 17.9 & 1.560 & 0.212 \\
\hline No & 10.8 & 23.2 & 24.5 & & \\
\hline Inductive protocols & & & & & \\
\hline Paclitaxel+platinum & 6.1 & 16.1 & 17.6 & 2.502 & 0.286 \\
\hline Gemcitabine+platinum & 0.0 & 7.7 & 7.7 & & \\
\hline Fluorouraci+platinum & 6.3 & 21.7 & 21.7 & & \\
\hline
\end{tabular}

Table 5. COX Regression Analysis on Influence of Each Factor on Metastatic Period

\begin{tabular}{|c|c|c|c|c|c|}
\hline \multirow[t]{2}{*}{ Influencing factors } & \multirow[t]{2}{*}{ Regression coefficient } & \multirow[t]{2}{*}{$\mathrm{P}$ value } & \multirow[t]{2}{*}{$\mathrm{RR}$} & \multicolumn{2}{|c|}{$95 \% \mathrm{CI}$} \\
\hline & & & & Lower limit & Upper limit \\
\hline T stages & 0.423 & 0.002 & 1.526 & 1.162 & 2.006 \\
\hline Induction protocols & & 0.088 & & & \\
\hline Induction protocol 1 & -0.577 & 0.038 & 0.561 & 0.326 & 0.968 \\
\hline Induction protocol 2 & -1.667 & 0.109 & 0.189 & 0.025 & 1.452 \\
\hline Induction protocol 3 & -0.161 & 0.658 & 0.851 & 0.417 & 1.737 \\
\hline Extracapsular invasion of lymph node & 0.544 & 0.050 & 1.723 & 0.999 & 2.970 \\
\hline
\end{tabular}

Relevance between metastasis and each factor

The metastatic rates of females, and of patients in phase IV and those with retropharyngeal lymph node and extracapsular invasion of lymph node were relevantly higher; the larger the $\mathrm{T}$ stages, the higher the metastatic rate; the metastatic rate was the highest in patients with superclavical lymphadenovarix, followed by those with lower-neck and upper-neck lymphadenovarix, and the rate of those without lymphadenovarix was the lowest; it was higher in patients without lost to follow-up than those with; it was higher in patients died than those survival and lost to follow-up; and there were significant differences in above comparison (Table 3). However, the metastatic rates of the rest factors had no significant difference (Table 3).

Influence of each factor on metastatic period

The life table method was applied to analyze the follow-up data of all patients, and the results indicated that the 1-, 3- and 5-year metastatic rates of LANPC patients were $6.6 \%, 17.5 \%$ and $18.8 \%$ respectively, with 
the average recurrent period being 73 months (95\% confidence intervals: 70.87 75.21 months).

In order to observe the influence of each factor on the metastatic period, log-rank test was adopted, in which $\mathrm{T}$ stages, $\mathrm{N}$ stages, radiotherapeutic techniques, concurrent chemotherapy, chemotherapeutic cycles and recurrence were excluded from the analysis due to their values, while the rest factor analysis results were shown in Table 4, which demonstrated that genders, $\mathrm{N}$ stages, clinical patterns, locations of lymphadenovarix, retropharyngeal lymph node, extracapsular invasion of lymph node and death had significant impact on the metastatic period of LANPC patients: the metastatic rates of females, and of patients in phase IV and those with retropharyngeal lymph node and extracapsular invasion of lymph node were relevantly higher; the larger the T stages, the higher the metastatic rate; the metastatic rate was the highest in patients with superclavical lymphadenovarix, followed by those with lower-neck and upper-neck lymphadenovarix, and the rate of those without lymphadenovarix was the lowest; it was higher in patients without lost to follow up than those with; it was higher in patients died than those survival and lost to follow up; and there were significant differences in above comparison; but the rest factors had no connection with the metastatic period.

On the basis of univariate analysis, genders, ages, pathological patterns, $\mathrm{T}$ stags, $\mathrm{N}$ stags, radiotherapeutic techniques, concurrent chemotherapy, locations of lymphadenovarix, induction chemotherapeutic protocols, retropharyngeal lymph node, chemotherapeutic cycles, extracapsular invasion of lymph node and metastasis were analyzed by multivariate analysis with $\mathrm{COX}$ risk ratio regression models (clinical stages were excluded from the analysis due to the high association with TNM stages). According to the COX regression analysis results, $\mathrm{T}$ stages, induction protocols and extracapsular invasion of lymph node were the independent prognostic factors for the metastasis of LANPC, and the metastasis was positively associated with $\mathrm{T}$ stages and extracapsular invasion of lymph node, which meant that $\mathrm{T}$ stages and extracapsular invasion of lymph node were the pestilent factors while reduction protocols the protective factor, and that the application of reduction protocols could greatly reduce the metastatic rates of LANPC patients (Table 5).

\section{Discussion}

With the continuous promotion of imaging technical techniques, improvement of radiotherapeutic techniques and the combined administration of radio-and chemotherapies, the survival rates of LANPC patients have been greatly increased. However, because LANPC patients are easy to have recurrence and metastasis, so recurrence and metastasis are still the major factors contributing to the post-radiotherapy death of LANPC patients (Dizman et al., 2014). Therefore, how to further control the therapeutic failure is of great significance. In addition, analysis on the clinical characteristics and the independent prognostic factors influencing the recurrence or metastasis of LANPC patients can devote contributions to seeking for more effective therapeutic methods for the decrease of recurrence or metastasis.

The post-therapeutic recurrence/metastasis involves multiple factors including biological characteristics, clinical stages and therapeutic protocols, etc. the present studies believed that the recurrence was closely correlated with clinical stages in LANPC patients, and the recurrent rate was higher in advanced LANPC patients after initial comprehensive treatment ( $\mathrm{Li}$ et al., 2012). Wang et al analyzed the factors with potential influences on the post-radiochemotherapeutic local control rate (LCR) of LANPC patients, in which the univariate analysis results revealed that the skull base bone destruction, $\mathrm{T}$ stages and pathological patterns diagnosed with MRI had significant impact on the LCR, and further COX regression analysis indicated that skull base bone destruction and pathological patterns diagnosed with MRI were the independent prognostic factors of the postradiochemotherapeutic LCR of LANPC patients (Wang et al., 2014). Additionally, another study pointed out that the cranial nerve involvement and tumor response were closely connected with the distant metastatic rate, while ages, cranial nerve involvement, $\mathrm{T}$ stages and tumor response were the prognostic factors impacting the DMFS and disease-free survival time of LANPC patients (Demirci et al., 2011).

This study analyzed the clinical data of 604 patients initially diagnosed as LANPC without distant metastasis, and the results found that recurrence was in correlation with clinical stages and $\mathrm{T}$ stages, and further analysis on the influence of each factor on the metastatic period suggested that the clinical stages had obvious impact on the metastatic period of LANPC. However, there was no significant difference in COX regression equation after analysis, demonstrating that the factors selected in this study were not the independent prognostic factors influencing the recurrence, which was predicated to be associated with the fact that the application of IMRT and the comprehensive chemotherapies might lead to discrepant LCR to LANPC patients. The relevance analysis between each factor and the metastasis of cancerous cells showed that ages, clinical stages, retropharyngeal lymph nodes, extracapsular invasion of lymph node, $\mathrm{N}$ stages and locations of lymphadenovarix were in relation with the metastasis of cancerous cells, and further univariate and multivariate analysis about the influence of each factor on the metastasis of LANPC prompted that $\mathrm{T}$ stages, induction protocols and extracapsular invasion of lymph node were the independent prognostic factors for the metastasis of LANPC, and the metastasis was positively associated with $\mathrm{T}$ stages and extracapsular invasion of lymph node and negatively connected with induction protocols, which meant that $\mathrm{T}$ stages and extracapsular invasion of lymph node were the pestilent factors while induction protocols the protective factor, and that the application of induction chemotherapy could greatly reduce the metastatic rates of LANPC patients. There was significant difference in the recurrent and metastatic periods between patients with and without lost to follow up, which was predicated to be associated with the loss of data in this study. Moreover, this study also illustrated that the survival patients were significantly lower in recurrent 
rate than those died, and death had obvious influence on the recurrent and metastatic periods, indicating that recurrence and metastasis were the major factors for the post-therapeutic death of LANPC patients.

To sum up, post-therapeutic metastasis were closely associated with $\mathrm{T}$ stages and extracapsular invasion of lymph node and the application of induction chemotherapy could greatly reduce the metastatic rates and then provide great benefits to LANPC patients.

\section{Acknowledgements}

This study was supported by Key Clinical Specialty Discipline Construction Program of Fujian, P.R.C. We wish to thank all members and patients involved to make this work successful. This study was supported by the National Clinical Key Specialty Construction Program, P.R.C, and Key Clinical Specialty Discipline Construction Program of Fujian, P.R.C.

\section{References}

Chen JL, Huang YS, Kuo SH, et al (2013). Intensity-modulated radiation therapy for $\mathrm{T} 4$ nasopharygeal carcinoma. Strahlenther Onkol, 189, 1001-8.

Demirci S, Kamer S, Kara G, et al (2011). Does the prognosis of nasopharyngeal cancer differ among endemic and nonendemic regions? Acta Otolaryngol, 131, 852-60.

Dizman A, Coskun-Breuneval M, Altinisik-Inan G, et al (2014). Reirradiation with robotic stereotactic body radiotherapy for recurrent nasopharyngeal carcinoma. Asian Pac J Cancer Prev, 15, 3561-6.

Hu S, Du J, Li D, et al (2014). Mitochondrial DNA haplogroup confers genetic susceptibility to nasopharyngeal carcinoma in chaoshanese from guangdong, china. PLOS ONE, 9 , 87795.

Lee AW, Ng WT, Chan LLK, et al (2014).Evolution of treatment for nasopharyngeal cancer- Sucess and setback in the intensity-modulated radiotherapy era. Radiother Oncol, 110, 377-84.

Li JX, Lu TX, Huang Y, et al (2012). Clinical characteristics of recurrent nasopharyngeal carcinoma in high-incidence area. Scientific World J, 2012, 719754.

Li K, Lin GZ, Shen JC, et al (2014). Time trends of nasopharyngeal carcinoma in urban Guangzhou over a 12-year period (20002011): declines in both incidence and mortality. Asian Pac J Cancer Prev, 15, 9899-903.

Lin S, Pan J, Han L, Zhang X, et al (2009). Nasopharyngeal carcinoma treated with reduced-volume intensity-modulated radiation therapy: report on the 3-year outcome of a prospective series. Int J Radiat Oncol Biol Phys, 75, 1071-8.

Lin SJ, Pan JJ, Han L, et al (2014). Update report of nasopharyngeal carcinoma treated with reduced-volume intensity-modulated radiation therapy and hypothesis of the optimal margin. Radiother Oncol, 110, 385-9.

Qu S, Liang ZG, Zhu XD (2015). Advances and challenges in intensity-modulated radiotherapy for nasopharyngeal carcinoma. Asian Pac J Cancer Prev, 16, 1687-92.

Wang Y,Zhao H, Zhang ZQ, et al (2014). MR imaging prediction of local control of nasopharyngeal carcinoma treated with radiation therapy and chemotherapy. $\mathrm{Br} J$ Radiol, 87, 20130657.

Wu F, Wang RS, Lu HM, et al (2014). Concurrent chemoradiotherapy in the locoregionally advanced nasopharyngeal carcinoma: Treatment outcomes of a prospective, multicentric clinical study. Radiother Oncol, 112, 106-11.

Wu SY, Wu YH, Yang MW, et al (2014). Comparison of concurrent chemoradiotherapy versus neoadjuvant chemotherapy followed by radiation in patients with advanced nasopharyngeal carcinoma in endemic area: experience of 128 consecutive cases with 5 year follow-up. Plos One, 14, 787.

Xu L, Pan J, Wu J, et al (2010). Factors associated with overall survival in 1706 patients with nasopharyngeal carcinoma: significance of intensive neoadjuvant chemotherapy and radiation break. Radiother Oncol, 96, 94-9.

Zeng L, Tian YM, Sun XM, et al (2014). Intensity-modulated radiotherapy for stage IVA/IVB nasopharygeal carcinoma. Strahlenther Onkol, 190, 993-1000. 